\section{Passing messages downstream}

ONE of the successes of the past few years in neurophysiology has been the description of how neurones communicate with their neighbours, as they must in any properly functioning nervous system. Whether the medium of communication is strictly electrical (literally the movement of ions across a gap junction) or the release of a chemical transmitter at a synapse, the immediate consequence is to increase the permeability of the postsynaptic membrane to the ions principally involved, $\mathrm{Na}^{+}$and $\mathrm{K}^{+}$. In the membranes of postsynaptic cells at gap junctions, the normal channels of permeability are sufficient. In chemical transmission, other distinct characteristics are involved.
So much has been demonstrated unambiguously by the technique of patch recording developed by Neher and his colleagues $^{7}$ but has earlier been demonstrated by Katz and Miledi ${ }^{18}$, at least for those forms of signalling between neurones in which the presynaptic cell excites a positive response downstream.

\section{The neuromuscular junction}

At the neuromuscular junction (where the neurotransmitter is acetylcholine), exposure of the muscle cell to transmitter can be recognized by the opening of sodium and potassium channels for periods measured in tens of milliseconds. More recently, Neher and Steinbach have shown that the period for which the channels are open can be increased by the use of analogues of the transmitter.

That the consequences of such an increase of permeability should tend to excite the postsynaptic cell is easily understood. For if such a cell is even partially depolarized electrically, the flux of $\mathrm{Na}^{+}$and $\mathrm{K}^{+}$ions is increased.

Thus, to some degree, the effects of a neurotransmitter on the membranes of a postsynaptic cell mimic the state of affairs obtaining during strictly electrical depolarization. Provided that the increased permeability is sufficient to correspond to the threshold voltage at which a cell will spontaneously produce (and propagate along its length) an action potential (a spike of voltage, or a pulse of current), even a chemical signal from a presynaptic cell will cause the postsynaptic cell to fire.

There is, however, endless scope for subtlety in this interaction. If the effect of a

\title{
Electrical connections
}

WITH the excitement of the past several years at the discovery of new chemicals or classes of chemicals capable of effecting communication between neurones, people have tended to overlook the importance of strictly electrical communication between neurones. They should not. The 1920s controversy between Sir Henry Dale, who argued that synapses (the points at which neurones approach each other closely) are bridged by neurotransmitters, and Sir John Eccles, who argued that electrical current did the job, has been settled as a drawn game. (Ironically, Eccles gave in at about the time that Fatt and $\mathrm{Kat}^{15}$ provided conclusive evidence in his support.)

Both methods of communication are common. One of the surprises of the past year or so is that electrical communication has been demonstrated in the mammalian hippocampus, hypothalamus and olfactory cortex, mostly by electron microscopy but in some investigations by physiological means.

Two features of such an electrical junction are distinctive ${ }^{16}$. First, the distance between the outer surface of the two neurones is an order of magnitude smaller than that across the typical chemical synapse, and may be merely 2 $\mathrm{nm}(20 \AA)$. Second, electron micrographs suggest a visible gap in each of the membranes placed so that the cytoplasm of the two cells may be thought to be in direct communication.

Electrical synapses are not, however, simply holes in neighbouring neurones that happen more or less to coincide with each other. Largely on the basis of electron micrography, it appears that such gaps are spanned by well-ordered hexagonal structures made of molecules of a protein called (no doubt for want of a better name) connexin.

The basic constituent of connexin is a protein molecule of about 27,000 daltons, whose amino acid constitution is well on the way to being determined. Hexamers of connexin form dumbbellshaped structures which double up, end to end, form "connexons" that span the gap between neurones, and are themselves arranged in a roughly hexagonal pattern (see, for example, Unwin and Zampighi ${ }^{17}$ ).

Electrical synapses have the advantage of speed. A signal in the presynaptic cell is a pattern of positive charge travelling along the cell process at a speed determined primarily by the capacitance of the cell membrane and the electrical conductance along the process.

At electrical synapses, where it is supposed that the inter-cell electrical conductance is high compared with that across the membrane, there is no reason why the wave of depolarization should not be projected directly into the postsynaptic cell. Moreover, the process is fast and a signal can be transferred from one cell to another without being spread out in time or made less sharp.

In many of their roles, electrical junctions may be important because they allow two-way communication. Such reciprocal signalling may be necessary when groups of neurones are required to function in concert. The increased frequency of electrical communication in cold-blooded animals such as fish suggests that it may be an adaptation to low-temperature operation, when the rate of cell metabolism on which chemical signalling depends is reduced. But

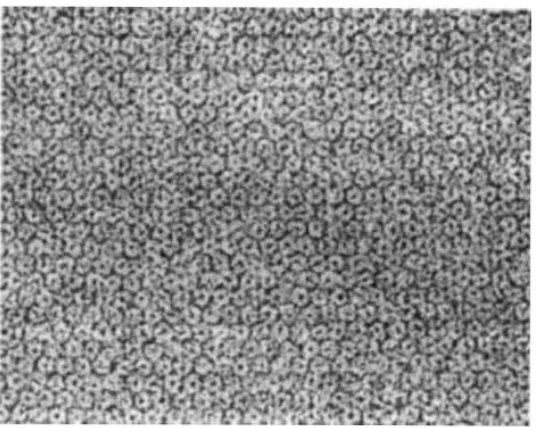

Fig. 6 Electron micrograph of isolated gap junction sheets viewed from a direction perpendicular to the plane of the membranes. The connexons are seen as annular units organized on a hexagonal lattice, with unit cell dimensions $85 \AA$. Uranyl acetate negative stain.

devotees insist that electrical signalling is not phylogenetically primitive - it does not occur in protozoa for example.

Moreover, it also seems that the efficiency of signalling across a gap junction is not simply a function of the shape of the gap, but that it can be regulated by physiological changes in the cell medium, especially by variations of $p \mathrm{H}$ and, less well, by the concentration of $\mathrm{Ca}^{2+}$. Those working in the field hold that the prevalence of electrical signalling between neurones is at least as important as the discovery of a new class of chemical neurotransmitters.

Indeed, electrical communication is surprisingly common. In the past year or so, gap junctions have been demonstrated in the mammalian hippocampus, hypothalamus and olfactory cortex, usually by means of electron microscopy but in some cases physiologically. Previously, electrical junctions had been recognized mostly in the peripheral nervous system - the spinal column, retina and so on. 